\title{
Review
}

\section{Inherited prothrombotic risk factors in children with first ischemic stroke}

\author{
Renata Zadro*, Désirée Coen Herak \\ Clinical Hospital Center Zagreb University School of Medicine, Department of Laboratory Diagnostics, Zagreb, Croatia \\ *Corresponding author: rzadro@mef.hr
}

\begin{abstract}
Stroke in children is a heterogeneous disorder. Over 100 risk factors for stroke have been reported and genetic predisposition to stroke has been established. The most frequently reported risk factors are congenital heart malformations, hemolytic anemias, collagen vascular diseases, some rare inborn metabolic disorders, trauma, infection and thrombophilia. The aim of this article is to provide an overview of investigated inherited prothrombotic risk factors in children with first ischemic stroke. Various prothrombotic risk factors have been investigated in pediatric stroke including elevated homocysteine and lipoprotein (a), antithrombin, protein C and protein S deficiency, Factor V Leiden, Factor II G20210A and plasminogen activator inhibitor-1 4G/5G polymorphism. Despite similar criteria for inclusion of different studies in meta-analyses investigating first ischemic stroke in children, the obtained results were not consistent for all prothrombotic risk factors. The discrepancies found could be explained by methodological issues like different sample sizes, patient populations included and lack of controls. In order to provide the necessary power for randomized control trials, multi-center, multi-national approaches like International Pediatric Stroke Study have been initiated with the aim to describe risk factors for childhood stroke and explore their relationship with presentation, age, geography, and infarct characteristics. Although it is evident from numerous studies that the frequency of inherited prothrombotic factors is increased in pediatric stroke, single thrombophilia does not fully explain stroke in a child as it represents only a mild risk factor. Further studies are needed, as improved understanding of underlying mechanisms will improve primary and secondary prevention of childhood stroke.
\end{abstract}

Key words: prothrombotic risk factors; childhood arterial ischemic stroke; ischemic perinatal stroke; cerebral sinovenous thrombosis

\section{Introduction}

Stroke is defined by the World Health Organization as rapidly developing signs of focal disturbance of cerebral function with symptoms lasting at least 24 hours or leading to death with no apparent cause other than of vascular origin (1). This definition is far from ideal for children, because children with symptoms compatible with transient ischemic attack can have brain infarction shown by brain imaging despite the transient nature of their symptoms. Consequently, pediatric stroke is defined as any neurological event including a seizure associated with an acute infarction shown by magnetic resonance imaging $(2,3)$.

Pediatric stroke is divided into ischemic and hemorrhagic stroke with subdivision of ischemic stroke according to the time of onset to childhood ischemic stroke and ischemic perinatal stroke (IPS). Ischemic stroke is also divided into arterial ischemic stroke
(AIS) and cerebral sinovenous thrombosis (CSVT). Classification of stroke is presented in Figure 1.

Childhood ischemic stroke is characterized by findings of the arterial- or venous- distribution ischemia in a child aged from the $29^{\text {th }}$ day postpartum to 18 years.

IPS is defined as a group of heterogeneous conditions characterized by focal disruption of cerebral blood flow secondary to arterial or cerebral venous thrombosis or embolization between 20 weeks of fetal life through the $28^{\text {th }}$ postnatal day, confirmed by neuroimaging or neuropathological studies (4). Because the timing of the vascular event leading to IPS is almost always unknown, it was suggested that classification of IPS should be based on gestational or postnatal age at diagnosis. Three subcategories have been suggested: (i) 


\section{PEDIATRIC STROKE}

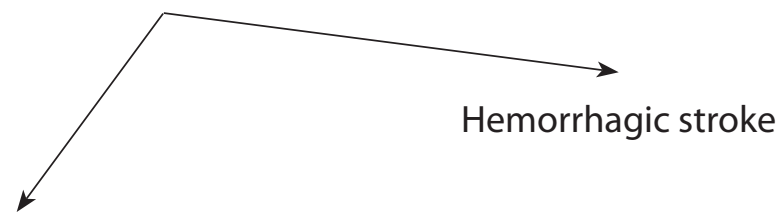

ISCHEMIC STROKE

CHILDHOOD

ischemic stroke

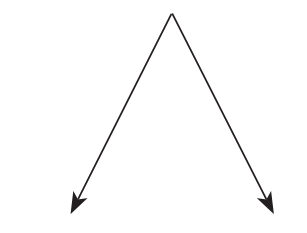

Arterial Venous

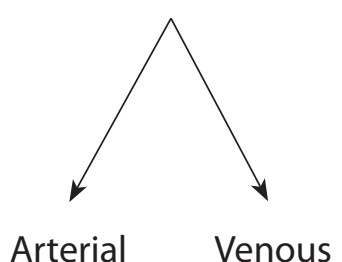

Arterial Venous

PERINATAL ischemic stroke

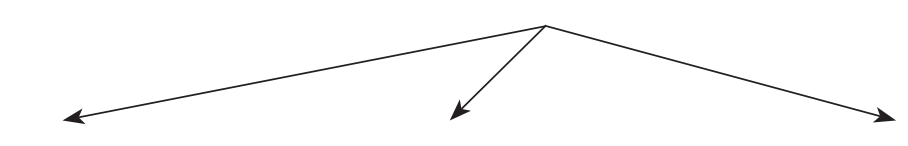

FETAL

ischemic

stroke

Figure 1. Classification of pediatric stroke.

fetal ischemic stroke diagnosed before birth by using fetal imaging methods; (ii) neonatal ischemic stroke diagnosed after birth and before $28^{\text {th }}$ postnatal day including preterm; (iii) presumed perinatal ischemic stroke, diagnosed in infants after the $28^{\text {th }}$ postnatal day in whom it is presumed that the event occurred between the $20^{\text {th }}$ week of fetal life through the $28^{\text {th }}$ postnatal day.

Although predominantly a disease occurring in adults, stroke is not uncommon in children and is increasingly recognized as an important cause of childhood disability and lifelong morbidity. There have been various population based studies of the incidence of pediatric stroke published over the last 30 years, with considerable variation in the reported incidence rates (rate per 100,000 children at risk per year). For overall ischemic and hemorrhagic stroke, reported incidence rate ranges from 1.3 to $13.0(5,6)$, with about half of the cases due to ischemia. The estimated incidence rate for CSVT is 0.67 while the incidence rate of childhood AIS ranges from 0.2 to 7.9, with increased frequency in black children and in boys $(7,8)$. The incidence of perinatal AIS ranges from 17 to 63 per 100,000 live births $(9,10)$.

Stroke in children is a heterogeneous disorder. Over 100 risk factors for stroke have been reported (Table 1) and genetic predisposition to stroke has been confirmed in animal models and in humans $(11,12)$. The most frequently reported risk factors are congenital heart malformations, hemolytic anemias, and collagen vascular diseases, as well as some rare inborn metabolic disorders, trauma, infection and thrombophilia (13-15). Despite the extensive investigation of potential risk factors, especially in last 10 years, no identifiable risk factor can be established in up to $30 \%$ of children (16).

Thrombophilia is an increased predisposition for the development of both arterial and venous thrombosis due to acquired or congenital abnormalities of the coagulation system $(17,18)$. The well established inherited prothrombotic risk factors for venous thrombosis are deficiency of natural anticoagulants, protein $C(P C)$, protein $S(P S)$ and antithrombin (AT), Factor V (FV) Leiden and Factor II 
TABLE 1. Risk factors for arterial ischemic stroke, cerebral sinovenous thrombosis and hemorrhagic stroke*

\begin{tabular}{|c|c|c|}
\hline Arterial ischemic stroke & Cerebral sinovenous thrombosis & Hemorrhagic stroke \\
\hline $\begin{array}{l}\text { Cardiac } \\
\text { - Congenital } \\
\text { - Acquired } \\
\text { - latrogenic }\end{array}$ & $\begin{array}{l}\text { General } \\
\text { - Dehydration } \\
\text { - Infection } \\
\text { - Fever } \\
\text { - Hypoxic-ischemic injury } \\
\text { - Post lumbar puncture }\end{array}$ & Genetic vasculopathy \\
\hline $\begin{array}{l}\text { Hematologic disorders } \\
\text { - Hemoglobinopathies } \\
\text { - Thrombophilia } \\
\text { - Iron deficiency anemia } \\
\text { - Thrombocytopenia }\end{array}$ & $\begin{array}{l}\text { Hematologic disorders } \\
\text { - Iron deficiency anemia } \\
\text { - Sickle cell disease } \\
\text { - Thalasemia } \\
\text { - Autoimmune hemolytic anemia } \\
\text { - Paroxysmal nocturnal hemoglobinuria } \\
\text { - Thrombophilia }\end{array}$ & $\begin{array}{l}\text { Hematologic disorders } \\
\text { - Hemoglobinopathies } \\
\text { - Platelet disorders } \\
\text { - Coagulopathy } \\
\text { - Hypofibrinogenemia }\end{array}$ \\
\hline $\begin{array}{l}\text { Infections } \\
\text { - Meningitis }\end{array}$ & $\begin{array}{l}\text { Head and neck disorders } \\
\text { - Infections } \\
\text { - Injury } \\
\text { - Hydrocephalus } \\
\text { - Post intracranial surgery }\end{array}$ & Trauma \\
\hline $\begin{array}{l}\text { Vasculitis } \\
\text { - Primary } \\
\text { - Secondary }\end{array}$ & Autoimmune disorders & $\begin{array}{l}\text { Hypertension } \\
\text { - Congenital } \\
\text { - Acquired }\end{array}$ \\
\hline Vasculopathies & Malignancy & \\
\hline $\begin{array}{l}\text { Other } \\
\text { - Trauma } \\
\text { - Toxin/Drugs } \\
\text { - Metabolic conditions }\end{array}$ & $\begin{array}{l}\text { Other } \\
\text { - Cardiac disease } \\
\text { - Renal disease } \\
\text { - Drugs } \\
\text { - Chromosomal disorders } \\
\text { - Metabolic conditions }\end{array}$ & \\
\hline
\end{tabular}

* based on references 11 and 12

(PT) G20210A (19). A brief summary of the most important factors and their role in hemostasis is presented in Table 2. Although various prothrombotic risk factors have been investigated in pediatric stroke, including elevation in homocysteine and lipoprotein (a) [Lp(a)], AT, PC and PS deficiency, FV Leiden and PT G20210A, they still represent one of the most confusing areas in childhood stroke literature. In this review article, data collected from numerous studies and meta-analyses on prothrombotic markers in childhood stroke are discussed.

\section{Childhood arterial ischemic stroke}

Published frequencies of inherited prothrombotic risk factors and magnitude of association in childhood AIS are shown in detail in Table 3.

Several studies have demonstrated the increased frequency of FV Leiden in childhood AIS as com- pared to healthy controls $(20-25,27,31,32,34,41)$. The most significant positive correlation between childhood AIS and FV Leiden (OR 6.0; 95\% Cl 3.0-12.1) has been found in a study with the largest number of AIS patients among case-control studies by German investigators (23), as well as in studies by Akar et al., Duran et al. and Herak et al. $(27,32,34)$.

The incidence of PT G20210A in children with AIS has also been investigated and included in a number of cohort and case-control studies (21$23,25,30,31,34,40,41)$ with controversial results.

At least two meta-analyses have been published assessing the association between childhood AIS and FV Leiden and PT G20210A $(40,41)$. While in a meta-analysis of Haywood et al. in 2005 (40), FV Leiden and PT G20210A were not associated with increased risk for chilhood AIS, Kenet et al. (41) revealed statistically significant association between 
TABLE 2. Pathophysiological background of presumed prothrombotic risk factors for childhood first ischemic stroke*.

\begin{tabular}{|c|c|}
\hline Prothrombotic factor & Pathophysiological relevance \\
\hline Factor V Leiden & $\begin{array}{l}\text { Single point mutation in FV gene causing resistance to proteolytic action of activated } \\
\text { protein C, leading to increased thrombin generation. }\end{array}$ \\
\hline Factor II G20210A & $\begin{array}{l}\text { Mutation in } 3^{\prime} \text { untranslated region of Fll gene associated with slightly increased plasma FII } \\
\text { levels resulting in hypercoagulable state. }\end{array}$ \\
\hline $\begin{array}{l}\text { Methylenetetra-hydrofolate } \\
\text { reductase C677T }\end{array}$ & $\begin{array}{l}\text { Mutation leading to mild decrease in enzymatic activity and increased homocysteine } \\
\text { level which is prothrombotic. }\end{array}$ \\
\hline Antithrombin deficiency & $\begin{array}{l}\text { Multifunctional serpin that inhibits acivated clotting enzymes (IIa, IXa, Xa, Xla) of the } \\
\text { coagulation pathway. }\end{array}$ \\
\hline Protein C deficiency & $\begin{array}{l}\text { Vitamin K-dependent protein with anticoagulant properties (proteolytic degradation of } \\
\text { FVa and FVIIla). }\end{array}$ \\
\hline Protein S deficiency & Cofactor of activated protein C, vitamin K-dependent. \\
\hline Lipoprotein (a) elevation & $\begin{array}{l}\text { Due to structural homology with plasminogen, it reduces plasmin generation by } \\
\text { competing with plasminogen binding to fibrin. }\end{array}$ \\
\hline $\begin{array}{l}\text { Plasminogen activator inhibitor-1 } \\
4 \mathrm{G} / 5 \mathrm{G} \text { polymorphism }\end{array}$ & $\begin{array}{l}\text { Polymorphism associated with elevated PAI-1 levels and an increased risk of } \\
\text { thromboembolic disease. }\end{array}$ \\
\hline Factor XIII-A Val34Leu & $\begin{array}{l}\text { Transglutaminase which mechanically stabilizes fibrin clot; in the presence of FXIII-A } \\
\text { Leu34 the formed fibrin is more susceptible to fibrinolytic degradation. }\end{array}$ \\
\hline $\begin{array}{l}\text { Human platelet alloantigen-1 } \\
\text { polymorphism }\end{array}$ & $\begin{array}{l}\text { Leu33Pro polymorphism of the } \beta_{3} \text { integrin gene which codes for the Illa subunit of the } \\
\text { GPIlb-Illa platelet receptor involved in platelet adhesion and thrombus formation. }\end{array}$ \\
\hline sed on reference 19. & \\
\hline
\end{tabular}

AIS and FV Leiden and PT G20210A (OR 3.7; 95\% Cl 2.8-4.8 and OR 2.6; 95\% Cl 1.7-4.1, respectively).

In children with first-episode stroke, only homozygous (TT) methylenetetra-hydrofolate reductase (MTHFR) C677T polymorphism has been shown to independently increase the risk of stroke $(23,37)$. The meta-analysis of Kenet et al. (41) demonstrated that the MTHFR C677T was found with higher frequency in childhood AIS than in healthy controls (OR 1.6; 95\% Cl 1.2-2.1). Although MTHFR C677T is generally considered to be relevant if serum homocysteine is elevated, homocysteine data were not available in the pediatric cohort examined in this meta-analysis.

Deficiencies of AT, PC and PS were also investigated in childhood AIS in a number of studies but only PC deficiency has been demonstrated as a risk factor for AIS in multiple case-control studies (23-25). The meta-analyses by Haywood et al. and Kenet et al. confirmed even stronger associations reported in the above studies $(40,41)$. As natural anticoagulant deficiencies can be transient, the potential limitation of the previously cited studies is that the data provided demonstrate an associa- tion between acquired thrombophilias and AIS only.

$L p(a)$ is a complex of low-density lipoprotein and apolipoprotein (a). Due to structural homology with plasminogen, $L p(a)$ inhibits plasmin generation by competing with plasminogen binding to fibrin. Lp(a) may also promote thrombosis through its lipoprotein (a) moiety by binding and inactivating tissue factor pathway inhibitor (42). Elevated Lp(a) has been identified as a genetically determined risk factor for stroke in young adults. Regarding its role as a risk factor for AIS in children, Lp(a) data are limited to three studies $(23,24,39)$ and one meta-analysis (41) that clearly showed association of elevated $\mathrm{Lp}(\mathrm{a})(>0.3 \mathrm{mg} / \mathrm{L})$ with presentation of AIS in white children.

The available data for other investigated prothrombotic risk factors [Plasminogen activator inhibitor-1 (PAI-1) 4G/5G, Factor XIII-A Val34Leu and Human platelet alloantigen-1 (HPA-1)] are not sufficient at the moment to make conclusions about their role as independent risk factors for childhood AIS $(28,29,35)$ although Biswas et al. (35) found association of HPA-1 and AIS in children. 
TABLE 3. Published frequencies of inherited prothrombotic risk factors and magnitude of association in childhood arterial ischemic stroke relative to healthy controls in different populations.

\begin{tabular}{|c|c|c|c|c|c|}
\hline Inherited thrombophilia & Population & Cases & Controls & OR $(95 \% \mathrm{CI})$ & Reference \\
\hline \multirow{15}{*}{ Factor V Leiden } & English & $6 / 50$ & $4 / 77$ & $2.3(0.6-8.6)^{1}$ & Ganesan (20) \\
\hline & Austrian & $6 / 22$ & $7 / 152$ & $4.6(1.2-17.2)$ & Zenz (21) \\
\hline & Turkish & $7 / 28$ & $10 / 106$ & $3.2(1.1-9.3)$ & Akar (22) \\
\hline & German & $30 / 148$ & $12 / 296$ & $6.0(3.0-12.1)$ & Nowak-Göttl (23) \\
\hline & German & $5 / 38$ & $4 / 100$ & $3.6(0.9-14.9)^{2}$ & Sträter (24) \\
\hline & Israeli & $10 / 58$ & $4 / 118$ & $4.8(1.4-16.5)$ & Kenet (25) \\
\hline & Turkish & $12 / 46$ & $3 / 68$ & $6.4(1.7-23.0)$ & Akar (27) \\
\hline & Argentinean & $1 / 44$ & $2 / 102$ & $1.2(0.2-13.2)$ & Bonduel (30) \\
\hline & Portuguese & $3 / 21$ & $4 / 115$ & $4.6(0.9-22.4)$ & Barreirinho (31) \\
\hline & Turkish & $7 / 30$ & $1 / 33$ & $9.7(1.1-452.3)$ & Duran (32) \\
\hline & Croatian & $4 / 33$ & $2 / 112$ & $7.6(1.3-43.5)$ & Herak (34) \\
\hline & Asian-Indian & $8 / 54$ & $10 / 58$ & $0.9(0.3-2.3)^{1,4}$ & Biswas (35) \\
\hline & Serbian & $1 / 26$ & $2 / 50$ & $1.0(0.1-11.1)$ & Djordjevic (36) \\
\hline & Meta-analysis & $71 / 629$ & $39 / 2004$ & $1.2(0.8-1.9)$ & Haywood (40) \\
\hline & Meta-analysis & $-/ 1014$ & $-/ 2581$ & $3.7(2.8-4.8)^{3}$ & Kenet (41) \\
\hline \multirow{10}{*}{ Factor II G20210A } & Austrian & $1 / 17$ & $1 / 98$ & $3.9(0.1-307.6)$ & Zenz (21) \\
\hline & Turkish & $5 / 28$ & $3 / 106$ & $7.4(1.7-33.5)$ & Akar (22) \\
\hline & German & $9 / 148$ & $4 / 296$ & $4.7(1.4-15.6)$ & Nowak-Göttl (23) \\
\hline & Israeli & $2 / 58$ & $3 / 118$ & $1.3(0.2-8.2)$ & Kenet (25) \\
\hline & Argentinean & $0 / 44$ & $1 / 102$ & / & Bonduel (30) \\
\hline & Portuguese & $2 / 21$ & $1 / 115$ & $11.8(1.0-136.5)$ & Barreirinho (31) \\
\hline & Croatian & $1 / 33$ & $4 / 112$ & $1.0(0.1-7.3)$ & Herak (34) \\
\hline & Serbian & $2 / 26$ & $3 / 50$ & $1.3(0.2-8.4)$ & Djordjevic (36) \\
\hline & Meta-analysis & $35 / 550$ & $15 / 1902$ & $1.1(0.5-2.3)$ & Haywood (40) \\
\hline & Meta-analysis & $-/ 1059$ & $-/ 2278$ & $2.6(1.7-4.1)^{3}$ & Kenet (41) \\
\hline \multirow{13}{*}{$\begin{array}{l}\text { Methylenetetra-hydrofolate } \\
\text { reductase C677T (only TT } \\
\text { homozygotes) }\end{array}$} & Turkish & $4 / 28$ & $6 / 106$ & $3.9(0.7-12.1)$ & Akar (22) \\
\hline & German & $30 / 148$ & $12 / 296$ & $2.6(1.5-4.5)$ & Nowak-Göttl (23) \\
\hline & Israeli & $8 / 58$ & $18 / 118$ & $1.1(0.4-2.7)$ & Kenet (25) \\
\hline & Spanish & $6 / 21$ & $4 / 28$ & $2.0(0.5-8.0)^{1}$ & Cardo (26) \\
\hline & Turkish & $4 / 46$ & $6 / 68$ & $1.0(0.3-3.7)^{1}$ & Akar (27) \\
\hline & Portuguese & $1 / 21$ & $13 / 115$ & $0.4(0.1-3.0)$ & Barreirinho (31) \\
\hline & Croatian & $5 / 33$ & $10 / 112$ & $1.8(0.6-5.8)$ & Herak (34) \\
\hline & Asian-Indian & $2 / 58$ & $0 / 58$ & $/ 4$ & Biswas (35) \\
\hline & Serbian & $1 / 26$ & $5 / 50$ & $0.4(0.04-3.3)$ & Djordjevic (36) \\
\hline & Polish & $9 / 64$ & $2 / 59$ & $5.8(1.0-42.7)$ & Zak (37) \\
\hline & USA & $4 / 15$ & $5 / 90$ & $1.1(0.2-4.0)$ & Morita (38) \\
\hline & Meta-analysis & $107 / 589$ & $127 / 1678$ & $1.7(1.2-2.3)^{5}$ & Haywood (40) \\
\hline & Meta-analysis & $-/ 777$ & $-/ 1715$ & $1.6(1.2-2.1)^{3}$ & Kenet (41) \\
\hline \multirow{5}{*}{ Antithrombin deficiency } & German & $0 / 148$ & $0 / 296$ & / & Nowak-Göttl (23) \\
\hline & German & $0 / 38$ & $0 / 100$ & / & Sträter (24) \\
\hline & Israeli & $0 / 58$ & $0 / 89$ & / & Kenet (25) \\
\hline & Meta-analysis & $1 / 435$ & $0 / 952$ & $1.0(0.3-3.7)$ & Haywood (40) \\
\hline & Meta-analysis & $-/ 639$ & $-/ 684$ & $3.3(0.8-15.5)^{3}$ & Kenet (41) \\
\hline \multirow{5}{*}{ Protein C deficiency } & German & $9 / 148$ & $2 / 296$ & $9.5(2.0-44.6)$ & Nowak-Göttl (23) \\
\hline & German & $6 / 38$ & $1 / 100$ & $18.5(2.1-16.0)^{2}$ & Sträter (24) \\
\hline & Israeli & $4 / 58$ & $1 / 89$ & $7.0(0.7-65.1)$ & Kenet (25) \\
\hline & Meta-analysis & $39 / 470$ & $11 / 1081$ & $6.5(3.0-14.3)$ & Haywood (40) \\
\hline & Meta-analysis & $-/ 844$ & $-/ 1207$ & $11.0(5.1-23.6)^{3}$ & Kenet (41) \\
\hline \multirow{5}{*}{ Protein S deficiency } & German & $0 / 148$ & $0 / 296$ & / & Nowak-Göttl (23) \\
\hline & German & $0 / 38$ & $0 / 100$ & / & Sträter (24) \\
\hline & Israeli & $0 / 58$ & $0 / 89$ & / & Kenet (25) \\
\hline & Meta-analysis & $13 / 428$ & $0 / 944$ & $1.1(0.3-3.8)$ & Haywood (40) \\
\hline & Meta-analysis & $-/ 574$ & $-/ 572$ & $1.5(0.3-6.9)^{3}$ & Kenet (41) \\
\hline
\end{tabular}


TABLE 3. (continued)

\begin{tabular}{|c|c|c|c|c|c|}
\hline Inherited thrombophilia & Population & Cases & Controls & OR $(95 \% \mathrm{CI})$ & Reference \\
\hline $\begin{array}{l}\text { Lipoprotein (a) elevation } \\
\text { (>0.3 } \mathrm{mg} / \mathrm{L})\end{array}$ & $\begin{array}{c}\text { German } \\
\text { German } \\
\text { Turkish } \\
\text { Meta-analysis }\end{array}$ & $\begin{array}{l}39 / 148 \\
7 / 38 \\
14 / 52 \\
-/ 616\end{array}$ & $\begin{array}{l}14 / 296 \\
5 / 100 \\
10 / 78 \\
-/ 578\end{array}$ & $\begin{array}{l}7.2(3.8-13.8) \\
4.3(1.3-14.4) \\
2.5(1.0-6.2) \\
6.5(4.5-9.6)^{3}\end{array}$ & $\begin{array}{l}\text { Nowak-Göttl (23) } \\
\text { Sträter (24) } \\
\text { Teber (39) } \\
\text { Kenet (41) }\end{array}$ \\
\hline $\begin{array}{l}\text { Plasminogen activator } \\
\text { inhibitor-1 4G/5G (only 4G4G } \\
\text { homozygotes) }\end{array}$ & $\begin{array}{l}\text { German } \\
\text { Turkish }\end{array}$ & $\begin{array}{c}65 / 198 \\
13 / 43\end{array}$ & $\begin{array}{l}275 / 951 \\
28 / 113\end{array}$ & $\begin{array}{l}1.2(0.9-1.7) \\
1.3(0.4-3.5)\end{array}$ & $\begin{array}{c}\text { Nowak-Göttl (28) } \\
\text { Akar (29) }\end{array}$ \\
\hline Factor XIII-A Val34Leu & Turkish & $25 / 116$ & $27 / 100$ & $0.8(0.4-1.5)^{1}$ & Akar (33) \\
\hline Human platelet alloantigen-1 & $\begin{array}{c}\text { Croatian } \\
\text { Asian-Indian }\end{array}$ & $\begin{array}{l}10 / 33 \\
18 / 58\end{array}$ & $\begin{array}{c}30 / 112 \\
6 / 58\end{array}$ & $\begin{array}{c}1.2(0.5-2.8) \\
3.0(1.1-8.1)^{1,4}\end{array}$ & $\begin{array}{l}\text { Herak (34) } \\
\text { Biswas (35) }\end{array}$ \\
\hline$\geq 2$ Genetic traits & Meta-analysis & $-/ 701$ & $-/ 1265$ & $18.7(6.5-54.1)^{3}$ & Kenet (41) \\
\hline \multicolumn{6}{|c|}{$\begin{array}{l}\text { OR - odds ratio; } \mathrm{Cl} \text { - confidence interval. } \\
{ }^{1} \mathrm{OR} \text { and corresponding } 95 \% \mathrm{Cl} \text { calculated by the present authors based upon data provided in the original report, }{ }^{2} \text { only children } \\
\text { with arterial ischemic stroke of cardiac origin; }{ }^{3} \text { pooled data of perinatal and childhood arterial ischemic stroke; }{ }^{4} \text { only children witl } \\
\text { non-cardioembolic arterial ischemic stroke; }{ }^{5} \text { pooled heterozygous and homozygous data. }\end{array}$} \\
\hline
\end{tabular}

\section{Ischemic perinatal stroke}

Ischemic stroke is 17 times more common in perinatal period than at any other time in childhood and adolescence. The pathogenesis of IPS is poorly understood but it may be due to a combination of changes in the maternal hemostasis system and thromboembolism from the placental side of the circulation. Besides the risk factors such as maternal health, pregnancy, delivery and family history for thrombotic events, congenital thrombophilic risk factors in the mother may aggravate the risk for thrombosis in the fetus (43).

In order to properly evaluate the importance of various prothrombotic factors in development of perinatal stroke, an extensive analysis was performed of the possible impact of prothrombotic factors in this disease entity. In Table 4, inherited prothrombotic risk factors associated with arterial IPS are summarized. In one prospective multicentre case control study (44), 62 out of 91 infants had at least one prothrombotic risk factor compared with 44 out of 182 controls (OR 6.7; 95\% Cl 3.8-11.6). The inherited prothrombotic abnormalities found were FV Leiden, PT G20210A, homozygous (TT) MTHFR and PC deficiency, and the most common abnormality was elevated Lp(a) observed in $22 \%$ of cases (OR 4.8; 95\% Cl 2.2-10.9). However, a cohort study of 35 neonates with stroke and 434 controls found a similar frequency of prothrom- botic risk factors in both groups except for PT G20210A but statistical significance was not reached (47).

Although the role of infant thrombophilia is extensively investigated, the role of maternal thrombophilia in the etiology of IPS has not been studied until recently. Two studies evaluating the biological profile of mother-child pairs with arterial IPS reported a higher prevalence of trombophilia in mothers than in children $(48,49)$. Mothers and children did not frequently (69\%) share the same prothrombotic risk factor or factors, which implies a possible contribution of paternal thrombophilia to perinatal ischemic thrombosis (48). In the study by Simchen et al. (48), of 23 mother-infant pairs, 12 (52\%) infants and 14 (61\%) mothers had genetic thrombophilia markers. Compared to healthy nulliparous pregnant women, mothers carrying FV Leiden increased 8.5-fold the risk for IPS in their children (95\% Cl, 4.1-17.5). The data presented suggest that understanding contribution of inherited prothrombotic factors, in both the mother and the child, is important but that the exact role of maternal thrombophilia in pathogenesis of IPS needs to be further evaluated.

\section{Cerebral sinovenous thrombosis}

Specific inherited prothrombotic risk factors that have been explored in childhood CSVT include 
TABLE 4. Published frequencies of inherited prothrombotic risk factors and magnitude of association in arterial perinatal ischemic stroke relative to healthy controls in different populations.

\begin{tabular}{|c|c|c|c|c|c|}
\hline Inherited thrombophilia & Population & Cases & Controls & OR $(95 \% \mathrm{CI})$ & Reference \\
\hline \multirow{9}{*}{ Factor V Leiden } & German & $17 / 91$ & $10 / 182$ & $3.9(1.7-9.0)$ & Günther (44) \\
\hline & German & $32 / 215$ & $10 / 182$ & $2.7(1.3-5.7)^{1}$ & Kurnik (45) \\
\hline & German & $19 / 76$ & $4 / 76$ & $5.8(1.7-20.1)$ & Debus (46) \\
\hline & USA & $1 / 35$ & $14 / 433$ & $0.9(0.1-6.9)^{1}$ & Miller (47) \\
\hline & \multirow{2}{*}{$\begin{array}{l}\text { Croatian } \\
\text { Israeli }\end{array}$} & $3 / 26$ & $2 / 112$ & $7.2(1.1-45.4)$ & Herak (34) \\
\hline & & $10 / 47$ & 7/112 & $3.4(1.2-9.5)^{1}$ & Simchen (48) \\
\hline & Estonian & $1 / 49$ & $12 / 400$ & $0.7(0.1-5.3)$ & Laugesaar (50) \\
\hline & Meta-analysis & n.a. & n.a. & $3.6(2.3-5.5)$ & Kenet (41) \\
\hline & Meta-analysis & $217 / 1328$ & $100 / 1863$ & $3.0(2.4-3.9)^{1,2}$ & Renaud (51) \\
\hline \multirow{9}{*}{ Factor II G20210A } & German & $4 / 91$ & $4 / 182$ & $2.0(0.4-8.3)$ & Günther (44) \\
\hline & German & $8 / 215$ & $4 / 182$ & $1.7(0.5-5.7)^{1}$ & Kurnik (45) \\
\hline & German & $4 / 76$ & $2 / 76$ & $2.3(0.3-17.2)$ & Debus (46) \\
\hline & USA & $2 / 35$ & $8 / 420$ & $3.0(0.6-14.7)^{1}$ & Miller (47) \\
\hline & Croatian & $0 / 26$ & $4 / 112$ & $0.5(0.1-8.7)$ & Herak (34) \\
\hline & Israelian & $3 / 47$ & $4 / 112$ & $1.8(0.4-8.3)^{1}$ & Simchen (48) \\
\hline & Estonian & $1 / 49$ & $13 / 400$ & $0.6(0.1-4.8)$ & Laugesaar (50) \\
\hline & Meta-analysis & n.a. & n.a. & $2.0(1.0-4.0)$ & Kenet (41) \\
\hline & Meta-analysis & $61 / 1157$ & $35 / 1442$ & $2.2(1.4-3.3)^{1,2}$ & Renaud (51) \\
\hline \multirow{6}{*}{$\begin{array}{l}\text { Methylenetetra-hydro- } \\
\text { folate reductase C677T } \\
\text { (only TT homozygotes) }\end{array}$} & German & $15 / 91$ & $20 / 182$ & $1.6(0.8-3.3)$ & Günther (44) \\
\hline & German & $9 / 76$ & $10 / 76$ & $0.6(0.2-2.4)$ & Debus (46) \\
\hline & USA & $4 / 35$ & $52 / 434$ & $1.0(0.3-2.8)^{1}$ & Miller (47) \\
\hline & Croatian & $4 / 26$ & $10 / 112$ & $1.9(0.5-6.5)$ & Herak (34) \\
\hline & Israeli & $9 / 47$ & $17 / 112$ & $1.3(0.5-3.0)^{1}$ & Simchen (48) \\
\hline & Meta-analysis & $-/ 777$ & $-/ 1715$ & $1.6(1.2-2.1)^{2}$ & Kenet (41) \\
\hline \multirow{4}{*}{ Antithrombin deficiency } & German & $0 / 91$ & $0 / 182$ & / & Günther (44) \\
\hline & German & $1 / 76$ & $0 / 76$ & / & Debus (46) \\
\hline & Israeli & $0 / 47$ & $0 / 112$ & / & Simchen (48) \\
\hline & Meta-analysis & $-/ 639$ & $-/ 684$ & $3.3(0.8-15.5)^{2}$ & Kenet (41) \\
\hline \multirow{4}{*}{ Protein C deficiency } & German & $6 / 91$ & $0 / 182$ & / & Günther (44) \\
\hline & German & $3 / 76$ & $0 / 76$ & / & Debus (46) \\
\hline & Israeli & $9 / 47$ & $2 / 112$ & $10.7(2.2-51.5)^{1}$ & Simchen (48) \\
\hline & Meta-analysis & $-/ 844$ & $-/ 1207$ & $11.0(5.1-23.6)^{2}$ & Kenet (41) \\
\hline \multirow{4}{*}{ Protein S deficiency } & German & $0 / 91$ & $0 / 182$ & / & Günther (44) \\
\hline & German & $0 / 76$ & $0 / 76$ & / & Debus (46) \\
\hline & Israeli & $6 / 47$ & $0 / 112$ & / & Simchen (48) \\
\hline & Meta-analysis & $-/ 574$ & $-/ 572$ & $1.5(0.3-6.9)^{2}$ & Kenet (41) \\
\hline \multirow{3}{*}{$\begin{array}{l}\text { Lipoprotein (a) elevation } \\
\text { (>0.3 } \mathrm{mg} / \mathrm{L})\end{array}$} & German & $20 / 91$ & $10 / 182$ & $4.8(2.2-10.9)$ & Günther (44) \\
\hline & German & $10 / 76$ & $6 / 76$ & $2.1(0.6-7.1)$ & Debus (46) \\
\hline & Meta-analysis & $-/ 616$ & $-/ 578$ & $6.5(4.5-9.6)^{2}$ & Kenet (41) \\
\hline $\begin{array}{l}\text { Plasminogen activator } \\
\text { inhibitor-1 4G/5G (only } \\
\text { 4G4G homozygotes) }\end{array}$ & USA & $7 / 35$ & $98 / 433$ & $0.6(0.3-1.3)^{1}$ & Miller (47) \\
\hline Human platelet & USA & $4 / 35$ & $102 / 434$ & $0.5(0.2-1.4)^{1}$ & Miller (47) \\
\hline alloantigen-1 & Croatian & $5 / 26$ & $30 / 112$ & $0.6(0.2-1.9)$ & Herak (34) \\
\hline$\geq 2$ Genetic traits & Meta-analysis & $-/ 701$ & $-/ 1265$ & $18.7(6.5-54.1)^{2}$ & Kenet (41) \\
\hline
\end{tabular}

OR - odds ratio; $\mathrm{Cl}$ - confidence interval; n.a. - not available.

${ }^{1} \mathrm{OR}$ and corresponding $95 \% \mathrm{Cl}$ calculated by the present authors based upon data provided in the original report; ${ }^{2}$ pooled data of perinatal and childhood arterial ischemic stroke. 
FV Leiden and PT G20210A, MTHFR C677T, AT, PC and PS deficiencies, Lp(a), PAI-1 4G/5G and HPA-1 polymorphism (Table 5). Two small pediatric casecontrol studies have examined the role of prothrombotic disorders in CSVT. Kenet et al. found prothrombotic condition in 19/46 children with CSVT, which was similar to the prevalence among 112 healthy controls (9). Heller et al. compared prothrombotic risk factors in 149 pediatric pa- tients with CSVT to 149 matched controls and found a significant association of CSVT with FV Leiden and Lp(a), PC and PS deficiencies (53). Strong association of FV Leiden (OR 12.9; 95\%Cl 2.3-73.0) and PT G20210A (OR 11.9; 95\%Cl 2.1-67.2) with CSVT was found in a study of Laugessar et al. (50). The meta-analysis that Laugessar et al. performed suggests that the risk for childhood CSVT is 3.1-fold increased both among FV Leiden and

TABLE 5. Published frequencies of inherited prothrombotic risk factors and magnitude of association in childhood cerebral sinovenous thrombosis relative to healthy controls in different populations.

\begin{tabular}{|c|c|c|c|c|c|}
\hline $\begin{array}{l}\text { Inherited } \\
\text { thrombophilia }\end{array}$ & Population & Cases & Controls & OR (95\% Cl) & Reference \\
\hline \multirow{8}{*}{ Factor V Leiden } & USA & $0 / 9$ & $2 / 65$ & / & Hagstrom (52) \\
\hline & German & $22 / 149$ & $8 / 149$ & $3.4(1.3-9.3)^{1}$ & Heller (53) \\
\hline & Argentina & $1 / 23$ & $2 / 102$ & $2.3(0.2-6.2)$ & Bonduel (30) \\
\hline & Israelian & $6 / 46$ & $7 / 112$ & $2.1(0.7-6.5)^{2}$ & Kenet (9) \\
\hline & USA & $2 / 24$ & $14 / 433$ & $2.6(0.5-12.0)^{2}$ & Miller (47) \\
\hline & Estonian & $2 / 7$ & $12 / 400$ & $12.9(2.3-73.0)$ & Laugesaar (50) \\
\hline & Meta-analysis & $-/ 1625$ & $-/ 2842$ & $2.7(1.7-4.3)$ & Kenet (41) \\
\hline & Meta-analysis & $33 / 258$ & $45 / 1261$ & $3.1(1.8-5.5)$ & Laugesaar (50) \\
\hline \multirow{7}{*}{ Factor II G20210A } & German & $7 / 149$ & $3 / 149$ & $3.8(0.8-17.3)^{1}$ & Heller (53) \\
\hline & Argentina & $1 / 23$ & $1 / 102$ & $4.6(0.3-76.3)$ & Bonduel (30) \\
\hline & Israelian & $2 / 46$ & $4 / 112$ & $1.2(0.2-6.9)^{2}$ & Kenet (9) \\
\hline & USA & $1 / 24$ & $8 / 420$ & $2.2(0.3-18.2)^{2}$ & Miller (47) \\
\hline & Estonian & $2 / 7$ & $13 / 400$ & $11.9(2.1-67.2)$ & Laugesaar (50) \\
\hline & Meta-analysis & $-/ 1409$ & $-/ 2613$ & $1.9(0.9-4.1)$ & Kenet (41) \\
\hline & Meta-analysis & $13 / 249$ & 29/1183 & $3.1(1.4-6.6)$ & Laugesaar (50) \\
\hline \multirow{3}{*}{$\begin{array}{l}\text { Methylenetetra-hydro- } \\
\text { folate reductase C677T } \\
\text { (only TT homozygotes) }\end{array}$} & Israeli & $2 / 46$ & $4 / 112$ & $1.2(0.2-6.9)^{2}$ & Kenet (9) \\
\hline & USA & $3 / 24$ & $52 / 434$ & $1.0(0.3-3.6)^{2}$ & Miller (47) \\
\hline & USA & $0 / 8$ & $5 / 90$ & I & Morita (38) \\
\hline \multirow{3}{*}{$\begin{array}{l}\text { Antithrombin } \\
\text { deficiency }\end{array}$} & German & $5 / 149$ & $0 / 149$ & / & Heller (53) \\
\hline & Israelian & $0 / 46$ & $0 / 112$ & I & Kenet (9) \\
\hline & Meta-analysis & $-/ 167$ & $-/ 469$ & 18.4 /3.2-104.3) & Kenet (41) \\
\hline \multirow{3}{*}{ Protein C deficiency } & German & $6 / 149$ & & $14.2(1.6-129.3)^{1}$ & Heller (53) \\
\hline & Israelian & $3 / 46$ & $\begin{array}{c}1 / 149 \\
2 / 112 / 1468\end{array}$ & $3.7(0.6-22.6)^{2}$ & Kenet (9) \\
\hline & Meta-analysis & $-/ 1031$ & $2 / 112 / 1468$ & $6.3(1.6-25.4)$ & Kenet (41) \\
\hline \multirow{3}{*}{ Protein S deficiency } & German & $8 / 149$ & $1 / 149$ & $17.0(1.9-151.2)^{1}$ & Heller (53) \\
\hline & Israelian & $1 / 46$ & $0 / 112$ & / & Kenet (9) \\
\hline & Meta-analysis & $-/ 187$ & $-/ 369$ & $5.3(1.5-18.2)$ & Kenet (41) \\
\hline $\begin{array}{l}\text { Lipoprotein (a) } \\
\text { elevation (>0.3 mg/L) }\end{array}$ & German & $44 / 106$ & $17 / 149$ & $7.2(3.7-14.2)^{1}$ & Heller (53) \\
\hline $\begin{array}{l}\text { Plasminogen activator } \\
\text { inhibitor-1 4G/5G (only } \\
\text { 4G4G homozygotes) }\end{array}$ & USA & $5 / 24$ & $98 / 433$ & $0.9(0.3-2.5)^{2}$ & Miller (47) \\
\hline $\begin{array}{l}\text { Human platelet } \\
\text { alloantigen-1 }\end{array}$ & USA & $8 / 24$ & $102 / 434$ & $1.4(0.6-3.2)^{2}$ & Miller (47) \\
\hline$\geq 2$ Genetic traits & Meta-analysis & $-/ 926$ & $-/ 1720$ & $6.1(0.9-43.1)$ & Kenet (41) \\
\hline \multicolumn{6}{|c|}{$\begin{array}{l}\mathrm{OR} \text { - odds ratio; } \mathrm{Cl} \text { - confidence interval. } \\
\text { 'univariate analysis; }{ }^{2} \mathrm{OR} \text { and corresponding } 95 \% \mathrm{Cl} \text { calculated by the present authors based upon data provided in the origina } \\
\text { report. }\end{array}$} \\
\hline
\end{tabular}


PT G20210A carriers (50). In contrast, in metaanalysis by Kenet et al. (41), that included up to 1625 CSVT patients and 2842 healthy controls, the association was demonstrated for FV Leiden only. In the same study Kenet et al. reported about the role of multiple thrombophilias as risk factors for CSVT that together with PT G20210A did not show a statistically significant association with first CSVT.

\section{Overview and limitations of published studies}

It is well known that the reliability of meta-analyses is dependent on inclusion of high-quality, methodologically similar studies. Numerous factors, e.g., sample sizes, patient populations included, proper selection of adequate controls, etc., have direct influence on reliability of results $(40,54)$. Despite similar criteria for the inclusion of different studies of childhood AIS in meta-analyses by Haywood et al. (40) and Kenet et al. (41), the obtained results were not consistent for all prothrombotic risk factors investigated. Both meta-analyses gave concordant results for majority of examined prothrombotic risk factors (AT, PC and PS deficiencies, MTHFR C677T). Discrepancies obtained for FV Leiden and PT G20210A results could be explained by the number of studies included in each meta-analysis ( 9 vs.17 and 7 vs.13, respectively), as well as by the number of patients and controls. In the above meta-analyses, a large amount of published data could not be included because of lack of controls; while in meta-analysis by Haywood et al. case-control studies were restricted to 4 countries (Germany, United Kingdom, Turkey and (srael), meta-analysis by Kenet et al. included additional countries (Austria, Portugal, Spain, North and South America). Moreover, the majority of studies were retrospective and not all inherited prothrombotic risk factors were tested in all patients. The same explanation can be applied for PT G20210A in CSVT patients in meta-analyses by Laugessar et al. (50) and Kenet et al. (41). Although a similar number of studies were included in both meta-analyses (6 and 5, respectively), the number of patients and controls was different. Furthermore, 3 case- control studies included in both meta-analyses originated from Argentina, Germany and Israel. Additional case-controls in the meta-analysis by Laugessar et al. (50), including their own, were from the United States (California and Philadelphia) while Kenet et al. (41) included 2 studies from Turkey.

For IPS, despite the fact that only 6 studies were available for the meta-analysis by Kenet et al. (41), summary ORs for FV Leiden (OR 3.6; 95\% Cl 2.35.5) and PT G20210A (OR 2.0; 95\% Cl 1.0-4.0) were similar to ORs obtained when pooled data of perinatal and childhood arterial ischemic stroke in the same meta-analysis were evaluated (OR 3.7; 95\% Cl 2.8-4.8 and OR 2.6; 95\% Cl 1.7-4.1, respectively). Furthermore, the obtained data are in concordance with results obtained in a meta-analysis by Renaud et al. (FV Leiden OR 3.0; 95\% Cl 2.4-3.9 and PT G20210A OR 2.2; 95\% Cl 1.4-3.3) (51). Although it is obvious that children with IPS are underrepresented in comparison with older children in the literature, the published results evaluating IPS only suggest that thrombophilia is still a risk factor.

Taking into consideration all the published studies, the study of Kenet et al. (41) is so far the most comprehensive meta-analysis of the effect of thrombophilia on pediatric stroke. It clearly shows persistent, strong association of FV Leiden and all types of stroke studied (OR 3.7; 95\% Cl 2.8-4.8 for first AIS onset and OR 2.7; 95\% CI 1.74.3 for first CSVT onset) resulting in summary OR of 3.3 (95\% Cl 2.6-4.1) for first AIS/CSVT onset. The association of PT G20210A and pediatric stroke (OR 2.6; 95\% Cl 1.7-4.1 for first AIS onset and OR 1.9; $95 \% \mathrm{Cl} 0.9-4.1$ for first CSVT onset) resulted in significant summary OR of $2.4(95 \% \mathrm{Cl}$ 1.7-3.5) for first AIS/CSVT onset. In contrast to weak association of MTHFR TT homozygosity with first AIS onset, such association was not demonstrated for CSVT. Meta-analysis by Kenet et al. (41) demonstrated the highest OR for combined genetic traits in first AIS and AIS/CSVT onset (OR 18.7; 95\% Cl 6.5-54.1 and OR 11.9; 95\% Cl 5.9-23.7, respectively). However, an unexpected finding was that $\geq 2$ genetic thrombophilia traits are less associated with CSVT than AT deficiency alone (OR 6.1; 95\% Cl 0.9-43.1 and OR 18.4; 95\% Cl 
3.2-104.3, respectively). The results of Kenet et al. highlight the fact that the ORs for AIS and CSVT differ with each thrombophilia, indicating distinct AIS and CSVT pathophysiologies. However, there are insufficient data in the article by Kenet et al. (41) or elsewhere in the literature to assess the possibly differing role of thrombophilia in these two subgroups.

\section{Perspectives}

Although a population-based cohort study is the superior study design for analyzing risk factors for a first stroke, ethnicity has a strong influence on prevalence of genetic thrombophilia and it is questionable whether the obtained results are applicable to other populations. Due to relative rarity of pediatric stroke, sufficient number of patients can only be achieved with multicenter, multi-national approaches in order to provide the necessary power for randomized control trials.

One prospective pediatric stroke registry is the International Pediatric Stroke Study (IPSS), the first international multi-center network of childhood stroke researchers from 30 centers in 10 countries. IPSS offers the advantage of rapid enrollment of patients through its web-based data entry where clinical presentations, risk factors, investigations, treatments and early outcomes are collected. IPSS was established in 2003 primarily to address the critical need to develop a set of standardized protocols to allow uniform collection of comprehensive data on the diagnosis (including laboratory testing), investigation, treatment and outcome assessment for all children with stroke. Until today 10 manuscripts have been published dealing with classification, risk factors and therapy of childhood AIS and neonatal AIS and CSVT (55-64).

One of the aims of IPSS is to describe the prevalence and spectrum of risk factors identified in children with AIS, to investigate:

a) whether the prevalence of risk factors varies by age and geographical region;

b) the association between a particular risk factor and mode of presentation; and c) the association between risk factors and infarct characteristics.

In the IPSS study by Kirton et al. (62), results of prothrombotic testing were inconsistent in 248 neonates with AIS. The presence of possible thrombophilia was reported in 47 (19\%) cases, including elevated Lp(a), homozygous (TT) MTHFR, FV Leiden, PT G20210A, PAI-1 4G/4G, AT and PS deficiency. Six children had multiple prothrombotic abnormalities. The authors conclude that their results do not contribute to the understanding of the role of thrombophilia in the etiology of neonatal AIS.

In the study by Mackay et al., 676 children with AIS across 30 centers were enrolled (63). Among 674 children tested, prothrombotic states were identified in 87 children (13\%), which was less frequent than in previous studies (65). Single prothrombotic risk factor was reported in 67 children and more than 1 prothrombotic risk factor in 20 children. After excluding acquired and not specified thrombophilia, 75 inherited prothrombotic risk factors were detected. There was a higher prevalence of prothrombotic risk factors in Europe compared to Asia, Australia and America. There was no statistical difference in prevalence of prothrombotic risk factors regarding age, mode of presentation and infarct characteristics. According to accumulated results so far IPSS has decided, as a next step, to continue research activities by dividing into working groups in order to collect an expanded data-set on selected stroke subtypes.

In conclusion, despite the proven evidence that the frequency of inherited prothrombotic factors is increased in children with stroke, a single thrombophilia does not fully explain a stroke in a child as it represents only a mild risk factor (ORs 4-10). Additional risk factors, especially arteriopathy, multiple thrombophilias or congenital heart disease are associated with increased ORs. Further studies are needed, as improved understanding of underlying mechanisms will improve the prospects for primary and secondary prevention of childhood stroke.

\section{Potential conflict of interest}

None declared. 


\section{References}

1. The World Health Organization MONICA Project (monitoring trends and determinants in cardiovascular disease): a major international collaboration. WHO MONICA Project Principal Investigators. J Clin Epidemiol 1988;41:105-14.

2. Seidman C, Kirkham F, Pavlakis S. Pediatric stroke: current developments. Curr Opin Pediatr 2007; 19:657-62.

3. Dlamini N, Kirkham FJ. Stroke and cerebrovascular disorders. Curr Opin Pediatr 2009;21:751-61.

4. Raju TN, Nelson KB, Ferriero D, Lynch JK, NICHD-NINDS Perinatal stroke workshop participants. Ischemic perinatal stroke: summary of a workshop sponsored by the National Institute of Child Health and Human Development and the National Institute of Neurological Disorders and Stroke. Pediatrics 2007;120:609-16.

5. Lynch JK, Hirtz DG, deVeber G, Nelson KB. Report of the National Institute of Neurological Disorders and Stroke workshop on perinatal and childhood stroke. Pediatrics 2002;109:116-23.

6. Agrawal N, Johnston SC, Wu YW, Sidney S, Fullerton HJ. Imaging data reveal a higher pediatric stroke incidence than prior US estimates. Stroke 2009;40:3415-21.

7. deVeber G, Andrew M, Adams C, Bjornson B, Booth F, Buckley DJ, et al. Cerebral sinovenous thrombosis in children. N Engl J Med 2001;345:417-23.

8. Laugesaar R, Kolk A, Uustalu Ü, Ilves P, Tomberg T, Talvik I, et al. Epidemiology of childhood stroke in Estonia. Pediatr Neurol 2010;42:93-100.

9. Kenet $G$, Waldman D, Lubetsky A, Kornbrut $N$, Khalil A, Koren $A$, et al. Paediatric cerebral sinus vein thrombosis: a multi-center, case-controlled study. Thromb Haemost 2004;92:713-8.

10. Laugesaar R, Kolk A, Tomberg T, Metsvaht T, Lintrop M, Varendi $\mathrm{H}$, et al. Acutely and retrospectively diagnosed perinatal stroke: A population based study. Stroke 2007;38:223440.

11. Lanni G, Catalucci A, Conti L, Di Sibio L, Paonessa A, Galluci M. Pediatric stroke: clinical findings and radiological approach. Stroke Res Treat 2011; doi:10.4061/2011/172168.

12. Dlamini N, Billinghurst L, Kirkham FJ. Cerebral venous (sinovenous) thrombosis in children. Neurosurg Clin N Am 2010;21:511-27.

13. Lanthier S, Carmant L, David M, Labrisseau A, deVeber G. Stroke in children: the coexistence of multiple risk factors predicts poor outcome. Neurology 2000;54:371-8.

14. Sträter R, Becker $S$, von Eckardstein A, Heinecke A, Gutsche $S$, Junker $R$, et al. Prospective assessment of risk factors for reccurent stroke during childhood - a 5-year follow-up study. Lancet 2002;360:1540-5.

15. Kirkham FJ, Prengler M, Hewes KM, Ganesan V. Risk factors for arterial ischemic stroke in children. J Child Neurol 2000;299-307.

16. Ganesan V, Prengler M, McShane MA, Wade AM, Kirkham FJ. Investigation of risk factors in children with arterial ischemic stroke. Ann Neurol 2003;53:167-73.

17. Moll S. Thrombophilias - practical implications and testing caveats. J Thromb Thrombolysis 2006;21:7-15.
18. de Moerloose P, Boehlen F. Inherited thrombophilia in arterial disease: a selective revue. Semin Hematol 2007;44:106 13.

19. Colman RW, Hirsch J, Marder VJ, Clowes AW, George JN, eds. Hemostasis and thrombosis: basic principles and clinical practice. 4th ed. Philadelphia, PA: Lippincott Williams and Wilkins, 2001.

20. Ganesan V, McShane MA, Liesnet R, Cookson J, Hann I, Kirkham FJ. Inherited prothrombotic states in ischaemic stroke in childhood. J Neurol Neurosurg Psychiatry 1998;65:508-11.

21. Zenz W, Bodo Z, Plotho J, Streif W, Male C, Bernert G, et al. Factor V Leiden and prothrombin gene $G 20210 \mathrm{~A}$ variant in children with ischemic stroke. Thromb Haemost 1998;80:763-6.

22. Akar N, Akar E, Deda G, Sipahi T, Orsal A. Factor V1691 G-A, prothrombin $20210 \mathrm{G}-A$, and methylenetetrahydrofolate reductase 677 C-T variants in Turkish children with cerebral infarct. J Child Neurol 1999;14:749-51.

23. Nowak-Göttl U, Sträter R, Heinecke A, Junker R, Koch HG, Schuierer $G$, et al. Lipoprotein (a) and genetic polymorphisms of clotting factor $V$, prothrombin, and methylenetetrahydrofolate reductase are risk factors of spontaneous ischemic stroke in childhood. Blood 1999;94:3678-82.

24. Sträter R, Vielhaber $H$, Kassenböhmer R, von Kries $R$, Göbel $U$, Nowak-Göttl U. Genetic risk factors of thrombophilia in ischaemicnchildhood stroke of cardiac origin: a prospective ESPED survey. Eur J Pediatr 1999;158(supp/ 3):S122-5.

25. Kenet G, Sadetzki S, Murad H, Martinowitz U, Rosenberg N, Gitel S, et al. Factor V Leiden and antiphospholipid antibodies are significant risk factors for ischemic stroke in children. Stroke 2000;31:1283-8.

26. Cardo E, Monrós E, Colomé C, Artuch R, Campistol J, Pineda $M$, et al. Children with stroke: polymorphism of the MTHFR gene, mild hyperhomocysteinemia, and vitamin status. $J$ Child Neurol 2000;15:295-8.

27. Akar N, Akar E, Ozel D, Deda G, Sipahi T. Common mutations at the homocysteine metabolism pathway and pediatric stroke. Thromb Res 2001;102:115-20.

28. Nowak-Göttl U, Sträter R, Kosch A, von Eckardstein A, Schobbes $R$, Luigs $P$, et al. The plasminogen activator inhibitor (PAI)-1 promoter $4 G / 4 G$ genotype is not associated with ischemic stroke in a population of German children. Childhood Stroke Study Group. Eur J Haematol 2001;66:57-62.

29. Akar N, Akar E, Yilmaz E, Deda G. Plasminogen activator Inhibitor-1 4G/5G polymorphism in turkish children with cerebral infarct and effect on factor $V$ 1691A mutation. $J$ Child Neurol 2001;16:294-5.

30. Bonduel M, Sciuccati G, Hepner M, Pieroni G, Torres AF, Mardaraz $C$, et al. Factor $V$ Leiden and prothrombin gene G20210A mutation in children with cerebral thromboem bolism. Am J Hematol 2003;73:81-6.

31. Barreirinho S, Ferro A, Santos M, Costa E, Pinto-Basto J, Sousa $A$, et al. Inherited and acquired risk factors and their combined effects in pediatric stroke. Pediatr Neurol 2003;28:134-8. 
32. Duran R, Biner B, Demir M, Celtik C, Karasalihoglu S. Factor $V$ Leiden mutation and other thrombophilia markers in childhood ischemic stroke. Clin Appl Thromb Haemost 2005; 11:83-8.

33. Akar N, Dönmez B, Deda G. FXIII gene Val34Leu polymorphism in Turkish children with cerebral infarct. J Child Neurol 2007;22;222-4.

34. Herak DC, Antolic MR, Krleza JL, Pavic M, Dodig S, Duranovic $V$, et al. Inherited prothrombotic risk factors in children with stroke, transient ischemic attack and migraine. Pediatrics 2009;123:e653-60.

35. Biswas A, Tiwari AK, Ranjan R, Meena A, Akhter MS, Yadav $B K$, et al. Prothrombotic polymorphisms, mutations, and their association with pediatric non-cardioembolic stroke in Asian-Indian patients. Ann Hematol 2009;88:473-8.

36. Djordjevic V, Stankovic M, Brankovic-Sreckovic V, Rakicevic $L j$, Radojkovic D. Genetic risk factors for arterial ischemic stroke in children: a possible MTHFR and eNOS gene-gene interplay? J Child Neurol 2009;24:823-7.

37. Zak I, Sarecka-Hujar B, Kopyta I, Emich-Widera E, Marszal E, Wendorff J, et al. The T allele of the 677C>T polymorphism of methylenetetrahydrofolate reductase gene is associated with an increased risk of ischemic stroke in Polish children. J Child Neurol 2009;24:1262-7.

38. Morita DC, Donaldson A, Butterfield RJ, Benedict SL, Bale JF. Methylenetetrahydrofolate reductase gene polymorphism and childhood stroke. Pediatr Neurol 2009;41:247-9.

39. Teber S, Deda G, Akar N, Soylu K. Lipoprotein (a) levels in childhood arterial ischemic stroke. Clin Appl Thromb Hemost 2010;16:214-7.

40. Haywood S, Liesner R, Pindora S, Ganesan V. Thrombophilia and first arterial ischaemic stroke: a systematic review. Arch Dis Child 2005;90:402-5.

41. Kenet G, Lutkhoff $L K$, Albisetti M, Bernard T, Bonduel M, Brandao $L$, et al. Impact of thrombophilia on arterial ischemic stroke or cerebral sinovenous thrombosis in children: a systematic review \& meta analysis of observational studies. Circulation 2010;121.1838-47.

42. Caplice NM, Panetta C, Peterson TE, Kleppe LS, Mueske CS, Kostner GM, et al. Lipoprotein (a) binds and inactivates tissue factor pathway inhibitor: a novel link between lipoproteins and thrombosis. Blood 2001;98:2980-7.

43. Cnossen $\mathrm{MH}$, van Ommen $\mathrm{CH}$, Appel IM. Etiology and treatment of perinatal stroke; a role for prothrombotic coagulation factors? Semin Fetal Neonatal Med 2009;14:311-7.

44. Günther G, Junker R, Sträter R, Schobess R, Kurnik K, Heller $C$, et al. Symptomatic ischemic stroke in full-term neonates: role of acquired and genetic prothrombic risk factors. Stroke 2000;31:2437-41.

45. Kurnik K, Kosch A, Sträter, R, Schobess R, Heller C, NowakGättl U, for the Childhood Stroke Study Group. Recurrent thromboembolism in infants and children suffering from symptomatic neonatal arterial stroke - a prospective follow-up study. Stroke 2003;34:2887-93.

46. Debus OM, Kosch A, Sträter R, Rossi R, Nowak-Göttl U. The factor V G1691A mutation is a risk for porencephaly: a case-control study. Ann Neurol 2004;56:287-90.
47. Miller SP, Wu YW, Lee J, Lammer EJ, lovannisci DM, Glidden $D V$, et al. Candidate gene polymorphisms do not differ between newborns with stroke and normal controls. Stroke 2006;37:2678-83.

48. Simchen MJ, Goldstein G, Lubetsky A, Strauss T, SchiffE, Kenet $G$. Factor $V$ Leiden and antiphospholipid antibodies in either mothers or infants increase the risk for perinatal arterial ischemic stroke. Stroke 2009;40:65-70.

49. Curry CJ, Bhullar S, Holmes J, Delozier CD, Roeder ER, Hutchison HT. Risk factors for perinatal arterial stroke: a study of 60 mother-child pairs. Pediatr Neurol 2007;37:99-107.

50. Laugesaar R, Kahre T, Kolk A, Uustalu U, Kool P, Talvik T. Factor V Leiden and prothrombin 20210G >A [corrected] mutation and paediatric ischaemic stroke: a case-control study and two meta-analyses. Acta Pediatr 2010;99:1168-74.

51. Renaud C, Tardy-Poncet B, Presles E, Chabrier S; AVCnn group. Low prevalence of coagulation F2 and F5 polymorphisms in mothers and children in a large cohort of patients with neonatal arterial ischemic stroke. Br J Haematol 2010;150:709-12.

52. Hagstrom JN, Walter J, Bluebond-Langner R, Amatniek JC, Manno CS, High KA. Prevalence of the factor $V$ Leiden mutation in children and neonates with thromboembolic disease. J Pediatr 1998;133:777-81.

53. Heller C, Heinecke A, Junker R, Knöfler R, Kosch A, Kurnik K, et al. Cerebral venous thrombosis in children-a multifactorial etiology. Circulation 2003;108:1362-7.

54. Simundic AM. Methodological issues of genetic association studies. Clin Chem Lab Med 2010;48:S115-8.

55. Sébire G, Fullerton $H$, Riou $E$, deVeber G. Toward the definition of cerebral arteriopathies of childhood. Curr Opin Pediatr 2004; 16:617-22.

56. Sofronas $M$, Ichord RN, Fullerton HJ, Lynch JK, Massicotte $M P$, Willan AR, et al. Pediatric stroke initiatives and preliminary studies. What is known and what is needed? Pediatr Neurol 2006;34:439-45.

57. Amlie-Lefond C, deVeber G, Chan AK, Benedict S, Bernard $T$, Carpenter J, et al. International Pediatric Stroke Study. Use of alteplase in childhood arterial ischemic stroke: a multicentre, observational cohort study. Lancet Neurol 2009;8:530-6.

58. Amlie-Lefond C, Bernard TJ, Sébire G, Friedman NR, Heyer GL, Lerner NB, et al. International Pediatric Stroke Study group. Predictors of cerebral arteriopathy in children with arterial ischemic stroke: results of the International Pediatric Stroke Study. Circulation 2009;119:1417-23.

59. Goldenberg NA, Bernard TJ, Fullerton HJ, Gordon A, deVeber G; International Pediatric Stroke Study group. Antithrombotic treatments, outcomes, and prognostic factors in acute childhood-onset arterial ischemic stroke: a multicentre, observational, cohort study. Lancet Neurol 2009;8:1120-7.

60. Golomb MR, Fullerton HJ, Nowak-Göttl U, deVeber G; International Pediatric Stroke Study group. Male predominance in childhood ischemic stroke: findings from the International Pediatric Stroke Study. Stroke 2009;40:52-7.

61. Jordan LC, Rafay MF, Smith SE, Askalan R, Zamel KM, deVeber $G$, et al. International Pediatric Stroke Study group. An- 
tithrombotic treatment in neonatal cerebral sinovenous thrombosis: results of the the International Pediatric Stroke Study. J Pediatr 2010;156:704-10.

62. Kirton A, Amstrong-Wells J, Chang T, deVeber G, Rivkin MJ, Hernandez $M$, et al. The International Pediatric Stroke Study investigators. Symptomatic neonatal arterial ischemic stroke. The International Pediatric Stroke Study. Pediatrics 2011:128:e1402-10.

63. Mackay TM, Wiznitzer M, Benedict SL, Lee KJ, deVeber GA, Ganesan V. Arterial ischemic stroke risk factors: the In- ternational Pediatric Stroke Study group. Ann Neurol 2011;69:130-40.

64. Bernard TJ, Manco-Johnson MJ, Lo W, Mackay MT, Ganesan $V$, deVeber $G$, et al. Towards a consensus-based classification of childhood arterial ischemic stroke. Stroke 2012;43:371-7.

65. Barnes $C$, deVeber $G$. Prothrombotic abnormalities in childhood ischemic stroke. Thromb Res 2006;118:67-74. 